\title{
Implementación de estrategia pedagógica mediada por las TIC para el fortalecimiento del pensamiento numérico en estudiantes de tercer grado de la Institución Educativa "El gas", San Pelayo, Córdoba.
}

\author{
Alba Luz Martínez Cogollo, Aleida Miranda Padilla \& Sandra Ruiz Beltrán \\ Maestría en Educación, Universidad Autónoma del Caribe, Barranquilla, Colombia \\ saje99@hotmail.com, aleidamirandap@hotmail.com, alvaluzmartinez@hotmail.com
}

Recibido: Noviembre 17, 2020.

Recibido en su versión corregida: Enero 24, 2021.

https://doi.org/10.54606/Sextante2021.v24.01

Aceptación: Febrero 07, 2021.

Cómo citar: Martínez Cogollo, A. L., Miranda Padilla, A., Ruiz Beltrán, S. (2021). Implementación de estrategia pedagógica mediada por las TIC para el fortalecimiento del pensamiento numérico en estudiantes de tercer grado de la Institución Educativa "El Gas", San Pelayo, Córdoba. Revista Sextante, 24, pp. 2 - 12, 2021.

\section{Resumen}

En este artículo se presentan los resultados de una investigación cuantitativa de tipo correlacional, que tuvo por objetivo proponer una estrategia pedagógica mediada por las TIC para el fortalecimiento del pensamiento numérico en estudiantes de grado tercero de la Institución Educativa "El Gas", municipio de San Pelayo, Córdoba, con el fin de mejorar el proceso de enseñanza-aprendizaje de las matemáticas y la calidad educativa en la misma. El estudio se desarrolló en 3 etapas: la primera consistió en la aplicación de una prueba diagnóstica para conocer el nivel del pensamiento numérico de los estudiantes de la institución, en la segunda se desarrolló la estrategia pedagógica MATE.TICS, utilizando la herramienta Classroom de Google. En la tercera etapa se realizó una prueba final para establecer el avance de los estudiantes gracias al impacto de la herramienta en el proceso de enseñanza-aprendizaje. En los resultados obtenidos se percibió una disminución del porcentaje de respuestas incorrectas, lo que permitió afirmar que la herramienta didáctica logró mejorar el indicador de resolución de problemas y la aplicación de operaciones matemáticas. Con todo lo expuesto anteriormente se concluye que, aunque existen varios aspectos por mejorar en el desarrollo del pensamiento matemático, sí se alcanzó la meta de presentar un avance por parte de los estudiantes, luego de la implementación de la estrategia didáctica.

Palabras clave: Aprendizaje, Herramienta didáctica, Pensamiento matemático, TIC.

\section{Implementation of a pedagogical strategy mediated by ICT for strengthening numerical thinking in third-grade students of "El Gas" School, San Pelayo, Córdoba.}

\begin{abstract}
This article presents the results of quantitative correlational research aimed at proposing a pedagogical strategy mediated by ICT to strengthen numerical thinking in third-grade students of the Educational Institution "El Gas", San Pelayo, Córdoba, to improve the mathematics teaching-learning process and the educational quality of the institution. The study was developed in 3 stages: the first consisted of applying an initial diagnostic test to know the level of numerical thinking of third-grade students of the institution. In the second stage, the pedagogical strategy MATE.TICS was developed using the Google Classroom tool. Finally, in the third stage, a final test was carried out to establish the students' progress thanks to the tool's impact on the teaching-learning process. The results obtained show a decrease in the percentage of incorrect answers, which allows us to affirm that the didactic tool improved the problem-solving indicator and the application of mathematical operations. In conclusion, there are several aspects to improve in the development of mathematical thinking, contributing by implementing the didactic strategy developed in this work to improve the students.
\end{abstract}

Keywords: Didactic tool; ICT; Learning; Mathematical Thinking. 


\section{Introducción}

La Agenda de Desarrollo Sostenible 2030 reconoce que la educación es el motor que impulsa el desarrollo de sociedades justas, equitativas y tolerantes, tras estipular que esta actúa como faro integrador para el alcance de los Objetivos de Desarrollo Sostenible (ODS) y ubicar la educación en ciencias, tecnologías, ingenierías y matemáticas (STEM) como los componentes que fomentan una educación de calidad; por ello, los gobiernos implementan estrategias para la consecución de los ODS en el marco de la promoción de oportunidades, que permitan a toda la población acceder a una educación acorde a sus necesidades y durante toda la vida (Organización de las Naciones Unidas, 2016).

En consecución, actualmente gran parte de los países que integran América Latina, adoptan los lineamientos internacionales en materia de calidad educativa, pero sus estadísticas revelan problemáticas en lo que respecta a los resultados que obtienen los estudiantes en las pruebas del Programa para la Evaluación Internacional de Alumnos -PISA, quienes, por lo general, no logran superar la media que estipula la Organización para la Cooperación y el Desarrollo Económico -OCDE$y$ reflejan un rezago en materia educativa en la región, especialmente en las áreas de ciencia y matemáticas. (OCDE, 2018). Como sustento de esto se tiene que países como México, República Dominicana y Perú ocupan las últimas posiciones de esta prueba al obtener los resultados más bajos del mundo, lo que constituye un desafío para las instituciones educativas, que frente a esta problemática se ven en la necesidad de implementar estrategias que faciliten el aprendizaje de los estudiantes y mejoren la calidad educativa en las áreas mencionadas (OCDE, 2018).

La panorámica anterior impulsa el desarrollo de investigaciones en el ámbito de las matemáticas, que evidencien la necesidad de fortalecer la relación de las TIC en los procesos de enseñanza-aprendizaje del área desde diversas perspectivas; particularmente, Hernández (2018), desarrolló un trabajo de grado que se trazó como objetivo revisar, seleccionar y organizar materiales tecnológicos para contribuir a la resolución de problemas matemáticos en los grados $3^{\circ}$ y $4^{\circ}$ de primaria, del cual concluyó que el material creativo de los ambientes virtuales es útil para los profesores, porque presenta actividades que facilitan el proceso de enseñanza y activan los aprendizajes. De igual forma se ve reflejado en el trabajo de Quinde y Rodríguez (2018), quienes aplicaron un programa de juegos lógicos en estudiantes de segundo grado de primaria, logrando un nivel destacado en actitud, aceptación, utilización y confianza en las matemáticas.

En lo que respecta a las competencias de los docentes de primaria se destaca el estudio de Morales (2015), quien determinó que la mayoría de los docentes no conoce otro tipo de recursos tecnológicos educativos distintos al computador de mesa, además, uno de cada diez docentes aprendió a utilizar las herramientas TIC en la universidad y solo $9,6 \%$ ha recibido algún tipo de capacitación por parte del Ministerio de Educación Pública (MEP) o por la institución educativa donde labora. Estos resultados le permitieron concluir que los esfuerzos y el presupuesto que el país destina al desarrollo de estas estrategias, no necesariamente se está traduciendo en capacitaciones pertinentes para los docentes.

Por otra parte, los resultados del análisis de documentos oficiales de la Institución Educativa "El Gas" del municipio de San Pelayo, Córdoba, tales como el Índice Sintético de la Calidad Educativa (ISCE), el cual permite a cada institución realizar un seguimiento de su progreso anual mediante los resultados de las Pruebas Saber, evaluando los componentes de desempeño, progreso, ambiente y eficiencia del 2018 (ver Figura 1), los resultados de la prueba SABER $3^{\circ}$ del 2017 (ver Figura 2 y Figura 3), y los informes generados por colegio del ICFES en el 2017 (ver Figura 4), permitieron identificar las debilidades que presentaban la institución, los estudiantes y docentes respecto al proceso de enseñanza-aprendizaje de las matemáticas, mostrando así un bajo rendimiento de los estudiantes pertenecientes al tercer grado de la institución educativa en el componente numérico variacional y en el geométrico métrico.

En el caso de las prácticas docentes de la Institución Educativa "El Gas", se puede afirmar que estas nutren el uso de metodologías tradicionales que privilegian el ejercicio mecánico de la repetición y la memorización. 


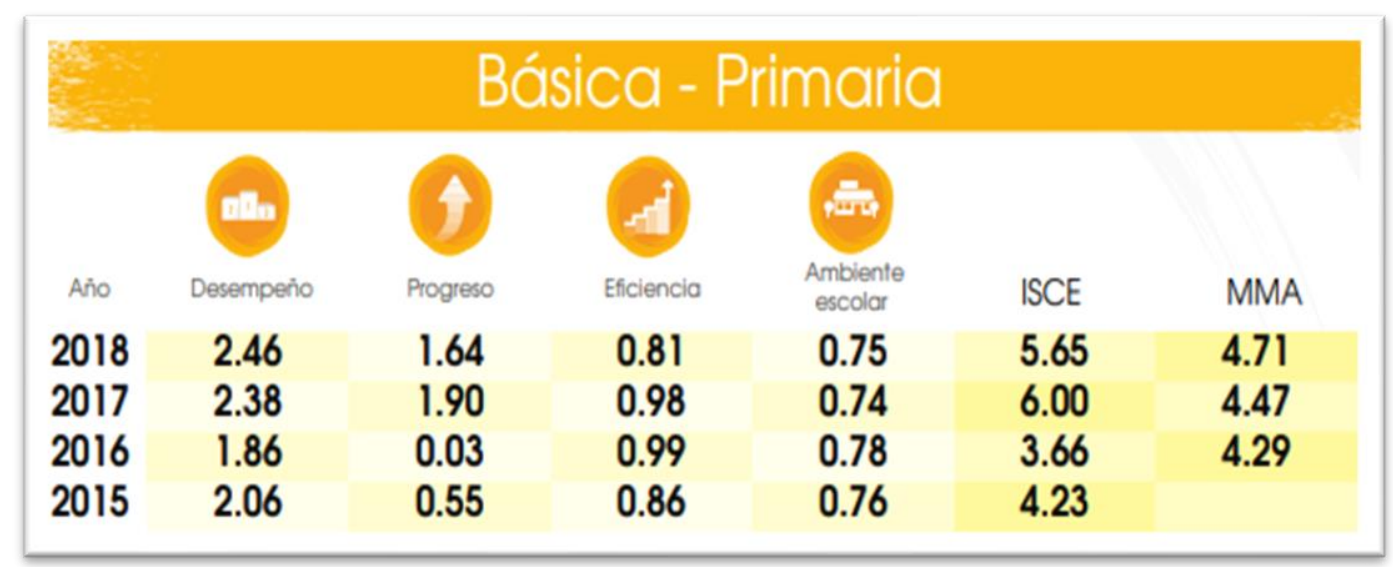

Figura 1. Reporte de la Excelencia de la Institución Educativa "El Gas" 2018, Córdoba Fuente: Ministerio de Educación Nacional, 2018.

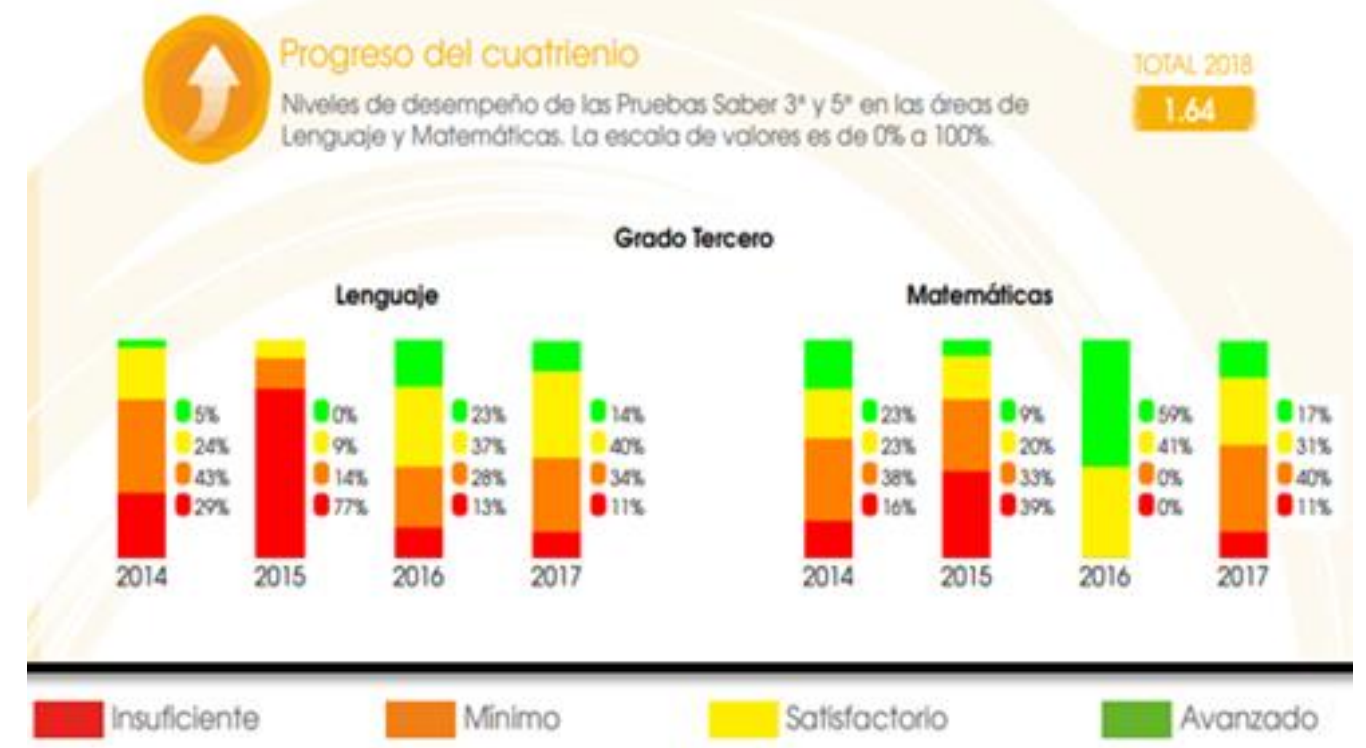

Figura 2. Progreso del Cuatrienio 2014-2017 de la Institución Educativa "El Gas".

Fuente: Ministerio de Educación Nacional, 2018.

\section{Básica - Primaria}

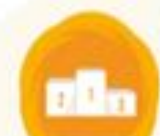

Desempeño del cuatrienio

Puntaje promedio de los Pruebas Saber $3^{\circ}$ y $5^{\circ}$ en las áreas de Lenguaje y Matemáticas. La escala de valores es de 100 a 500. siendo 500 el puntaje promedio más alto posible.

Lenguaje

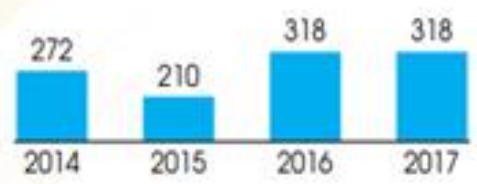

\section{Grado Tercero}

Matemáticas

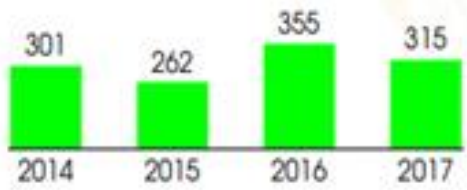

Figura 3. Desempeño del Cuatrienio 2014-2017 de la Institución Educativa "El Gas".

Fuente: Ministerio de Educación Nacional, 2018. 


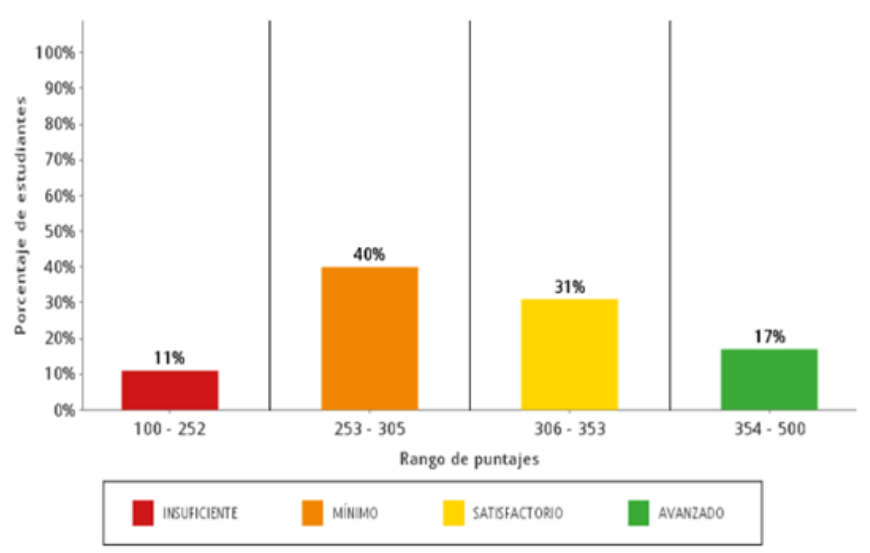

Figura 4. Porcentaje de Estudiantes por Niveles de Desempeño en la Institución Educativa “El Gas”, 2017.

Fuente: Ministerio de Educación Nacional, 2018.

De igual forma, el uso de herramientas tecnológicas se encontró muy limitado, ya que el plantel no cuenta con la logística necesaria para utilizar dichos recursos, aspecto que dificulta y limita el surgimiento de alternativas que favorezcan su aprovechamiento. A partir de los planteamientos anteriores, nació el siguiente interrogante de investigación: ¿Qué estrategia pedagógica, mediada por las TIC, se puede proponer para mejorar la competencia del pensamiento numérico en estudiantes de grado tercero, de la Institución Educativa "El Gas" del municipio de San Pelayo, Córdoba, Colombia?

Este interrogante orientador permitió desplegar la presente investigación, la cual es de tipo correlacional debido a que busca conocer la relación que existe entre dos o más categorías o variables en un contexto en particular, con el objetivo de proponer una estrategia pedagógica mediada por las TIC que permita potenciar el pensamiento matemático numérico en estudiantes de grado tercero de la institución mencionada, a partir de la identificación de herramientas didácticas medidas por las TIC, la valoración de las competencias del pensamiento numérico de los estudiantes de grado tercero y el aprovechamiento de la herramienta didáctica seleccionada.

El desarrollo de estrategias que potencien la comprensión y análisis de problemas matemáticos es un tema de importancia en la actualidad, ya que el reto de la educación es formar ciudadanos con pensamiento crítico y capacidad analítica, las cuales le permitan innovar y desarrollar competencias de manera creativa, por lo tanto, es fundamental atender tales aspiraciones desde la básica primaria, mediante el uso de herramientas tecnológicas que ubiquen al docente como facilitador y mediador del aprendizaje y al estudiante como protagonista del mismo.

Bajo estos argumentos, los resultados de esta investigación se verán reflejados en el mejoramiento de los resultados de las pruebas de los estudiantes de grado tercero de la Institución Educativa El Gas, en las competencias de razonamiento y resolución de problemas. De allí que la relevancia del presente estudio se fundamente en el aprendizaje de problemas matemáticos a partir del uso de una herramienta didáctica mediada por las TIC, resaltando de este modo, la intervención del docente y la motivación de los estudiantes como eje central de la intervención.

\section{Fundamentos teóricos}

La investigación se fundamenta teóricamente en tres conceptos vitales: la teoría conectivista del aprendizaje, la didáctica de las matemáticas y las TIC. En definición, el conectivismo es la integración de principios explorados por el caos, la red y las teorías de complejidad y auto organización (ver Figura 5). Permite interpretar los procesos relacionados a la forma como las personas aprenden $\mathrm{y}$ adquieren conocimientos en el mundo actual, enmarcado en la evolución tecnológica de las redes sociales y los ambientes multiformes de aprendizaje, donde estos procesos están relacionados a las tecnologías a través del elearning (Siemens, 2004).

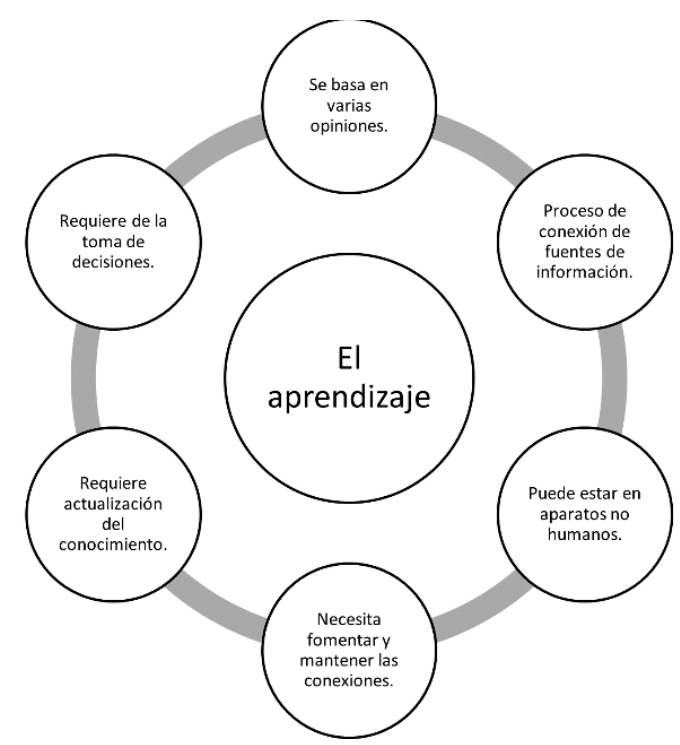

Figura 5. El aprendizaje según el Conectivismo. Fuente: Tomado y adaptado de Siemens (2004). 
Cabe resaltar que el conectivismo comparte la visión del estudiante como protagonista de su aprendizaje con el constructivismo, concediéndole un papel activo en la elección de la información y la forma de organizarla. No obstante, ambas teorías se diferencian en un aspecto muy importante que es la idea de la constante construcción de significados. El rol del docente en la formación, desde el enfoque del conectivismo, debe priorizar la enseñanza entre la relación presencial y digital, desde la combinación de diferentes materiales (auditivos, visuales y audiovisuales), mientras que el estudiante, específicamente el de la educación primaria, debe tener acompañamiento para el acceso a las distintas herramientas digitales a su alcance o facilitados por el docente (Siemens, 2006).

En adición a lo antes expuesto, otra posición con respecto a este concepto lo encontramos indicado por Giesbercht (2007), quien explica que el conectivismo se fundamenta en la interacción del estudiante con elementos que permiten experiencias en la vida real, llevando la práctica de aprendizaje fuera de las aulas de clase mediante las conexiones, manteniendo como principio esencial el holismo y el balance entre las necesidades de quienes aprenden y las de la institución.

Por su parte, en la didáctica de las matemáticas, son fundamentales las interacciones, intervenciones y fenómenos que se producen entre sus tres principales actores; el docente, el estudiante y el saber, siendo el primero quien se forma para transmitir el saber y poner en marcha el proceso de enseñanza de la manera más apropiada.

Sin embargo, es necesario incluir en este triángulo pedagógico a las interacciones didácticas, las cuales se centran en un objetivo instruccional que no es más que el objetivo educativo que se intenta alcanzar, mediante un discurso didáctico que busca enseñar a los estudiantes todo lo relacionado al objeto referente (del que hace referencia el discurso didáctico), el estudiante a su vez puede aprender del objeto referente mediante la observación y estudiar el discurso didáctico del que hace referencia dicho objeto (Ibáñez, 2007).

Ahora bien, en la educación primaria es oportuno afirmar que las TIC inciden de manera significativa, ya que poseen un papel considerable en el proceso de enseñanza-aprendizaje de las matemáticas si se utilizan apropiadamente, debido a que si esto no fuera así podrían dificultar el proceso, pasando de ser una gran herramienta para convertirse en una barrera del aprendizaje de los estudiantes. Aun así, las TIC se han ido generalizando en la cotidianidad educativa, transformado los métodos de enseñanza y constituyéndolos como un recurso de mucha utilidad para enseñar de forma lúdica, innovadora e interactiva la resolución de problemas matemáticos. (Real, 2013)

En lo que respecta a la Didáctica de las Matemáticas, las TIC proponen el replanteo por parte de los docentes, de sus planes de curso y metodologías pedagógicas elegidas, lo que no se considera como la solución a las dificultades que presenta el proceso de enseñanza-aprendizaje de las matemáticas, pero sí se puede afirmar que produce un cambio en la manera de enseñar. (Cruz y Puentes, 2012)

En Colombia la implementación de las TIC en el aula es un tema de creciente interés, reafirmándose en la Ley 1341 del 30 de julio de 2009, que define las TIC como el conjunto de Tecnologías de la Información y las Comunicaciones. Donde el Ministerio de Tecnologías de la Información y las Comunicaciones es el responsable de coordinar la articulación del plan TIC con el de Educación, apoyando al Ministerio de Educación Nacional para avanzar en los objetivos de implementación, fomento y utilización de las TIC en el aula. (MINTIC, 2009)

En este sentido, los recursos tecnológicos aplicados en el proceso didáctico de la matemática en la formación de la básica primaria. tienen la finalidad de iniciar al estudiante en el uso de las Tecnologías de la Información y la Comunicación, formando así alumnos capaces de resolver problemas y adaptándolos a la nueva sociedad de nativos digitales. Por ello, los alumnos al aprender matemáticas por medio de las TIC mejoran la comprensión, descubren por sí mismos conceptos y desarrollan un aprendizaje significativo, alcanzando las competencias deseadas.

\section{Metodología propuesta}

Esta investigación se desarrolla en el paradigma cuantitativo de tipo correlacional, el cual, según Hernández, Fernández y Baptista (2014), representa 
un conjunto de procesos secuenciales y probatorios que reflejan la necesidad de estimar magnitudes de los problemas de investigación, ya que se propone un problema de estudio concreto y delimitado. Para la recolección de los datos se utilizan procedimientos estandarizados y para su análisis se usaron métodos estadísticos, además, busca obtener y analizar datos cuantitativos sobre variables que fueron determinadas previamente.

La investigación se realizó durante los dos primeros periodos del año escolar 2020, en la Institución Educativa "El Gas" del municipio de San Pelayo, Córdoba. La muestra del estudio correspondió a los 30 estudiantes de tercer grado del plantel. Durante la primera etapa se elaboró y aplicó una prueba diagnóstica, utilizando 15 preguntas de la estrategia Supérate con el Saber, para el grado $3^{\circ}$ del año 2017, lo cual permitió conocer el nivel de estos estudiantes en la competencia del pensamiento numérico, previo a la aplicación de la estrategia pedagógica mediada por las TIC.

Partiendo del punto de referencia brindado por los resultados de la prueba diagnóstica, se pudo desarrollar una plataforma LSM o Sistema de
Gestión del Aprendizaje a través de la herramienta Classroom, de Google. Esta se llamó MATE.TICS (ver Figura 6), para fortalecer la competencia del pensamiento numérico en los estudiantes de tercer grado de la misma institución. Esta herramienta fue aplicada durante 3 meses y posterior a este periodo se aplicó una prueba final, utilizando 15 preguntas del cuadernillo de la prueba Saber $3^{\circ}$ relacionada al pensamiento numérico. Finalmente, se compararon los resultados obtenidos en ambas pruebas para conocer el impacto de esta herramienta en el proceso de enseñanza-aprendizaje de la asignatura de matemáticas.

\section{Resultados y análisis}

4.1. Primera fase: Prueba diagnóstica inicial

La prueba diagnóstica aplicada durante la primera fase de la investigación permitió conocer el nivel de conocimiento de los estudiantes en cuanto a la competencia del pensamiento numérico, arrojando los resultados representados en las Figuras $\underline{7}, \underline{8}$ y $\underline{9}$, los cuales describen dicha información en función del porcentaje de respuestas incorrectas por pregunta.

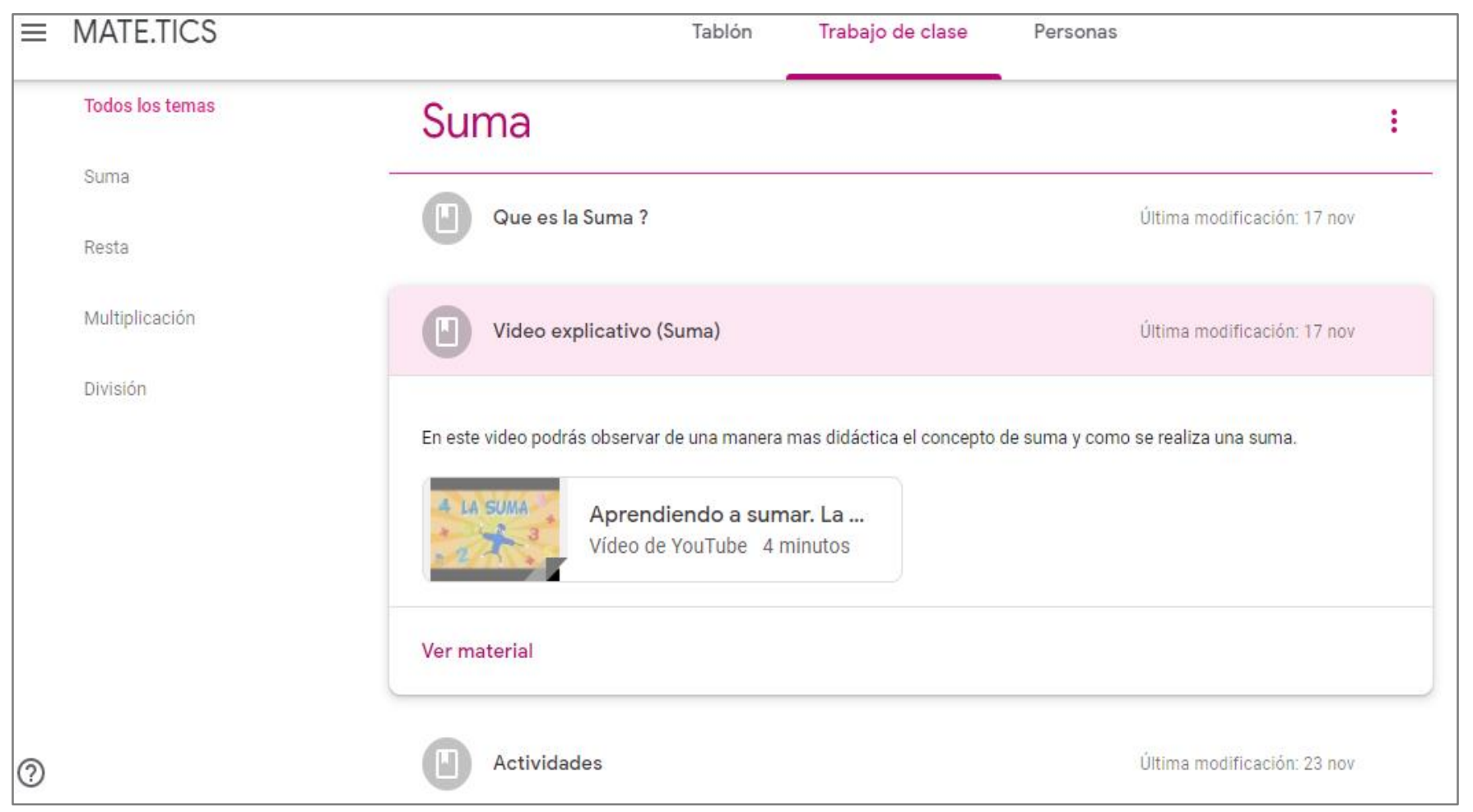

Figura 6. Interfaz gráfica de la herramienta MATE.TICS.

Fuente: Imagen tomada de la herramienta MATE.TICS. 
Los resultados arrojaron que existe un problema por parte de la población en el área de matemáticas, ahora bien, el $46 \%$ de los estudiantes acertaron a las respuestas correctas, demostrando que existe un problema en el proceso de enseñanza-aprendizaje.

Adicionalmente, los resultados obtenidos por los estudiantes en la primera prueba diagnóstica muestran que menos del $50 \%$ respondió acertadamente el $66.7 \%$ de las preguntas, y que en solo dos preguntas más del $60 \%$ escogieron la respuesta correcta, lo que permite afirmar que existen deficiencias en la competencia del pensamiento numérico.

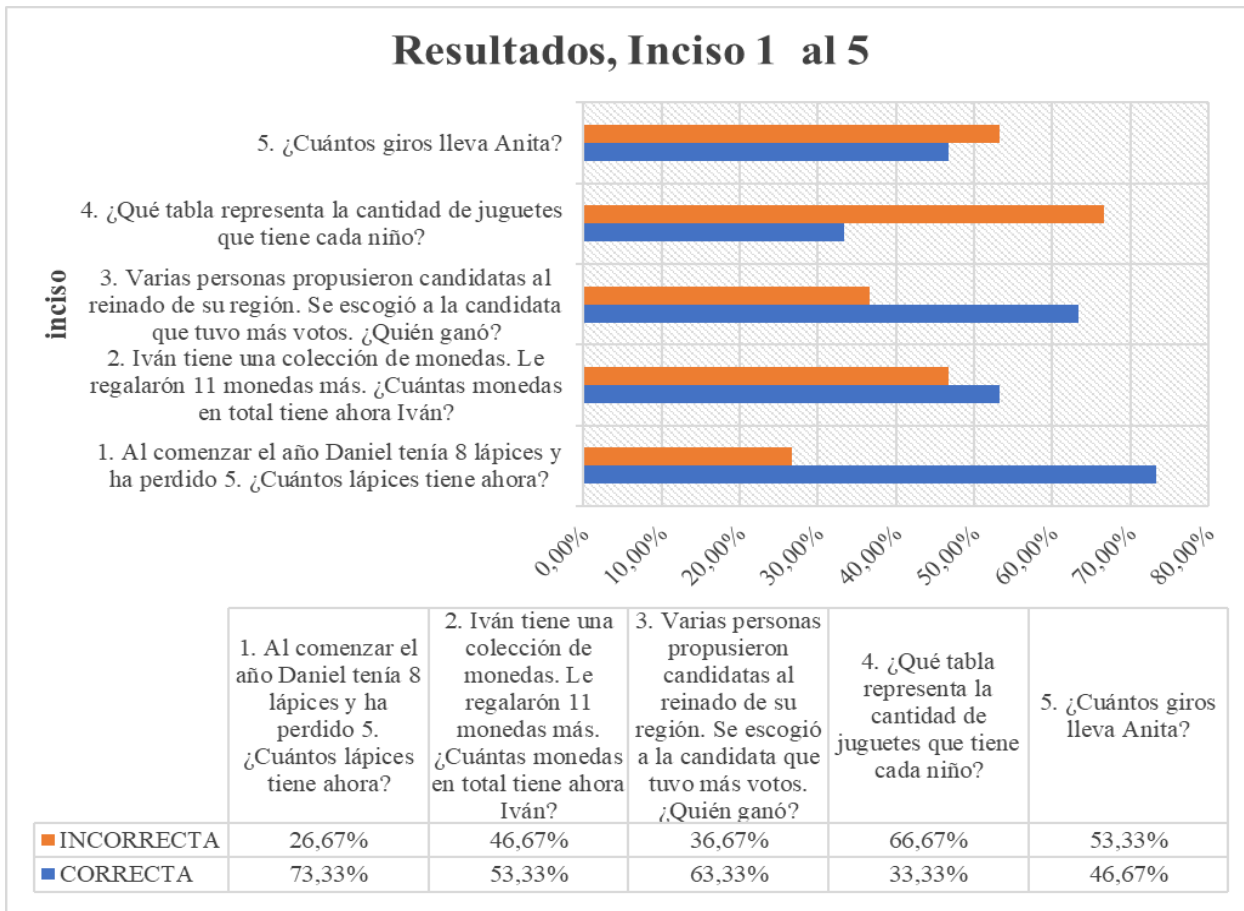

Figura 7. Resultados prueba diagnóstica, incisos del 1 al 5.

Fuente: Los autores.

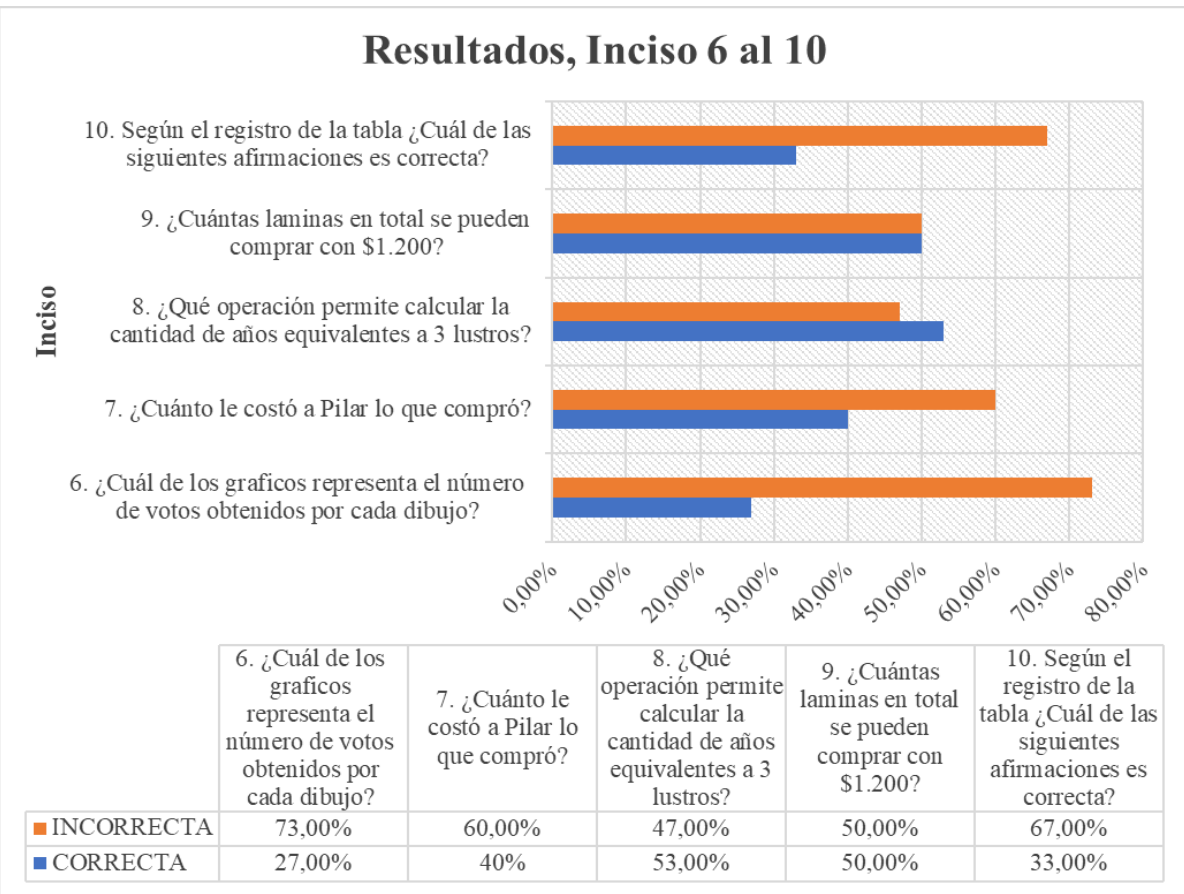

Figura 8. Resultados prueba diagnóstica, incisos del 6 al 10.

Fuente: Los autores. 


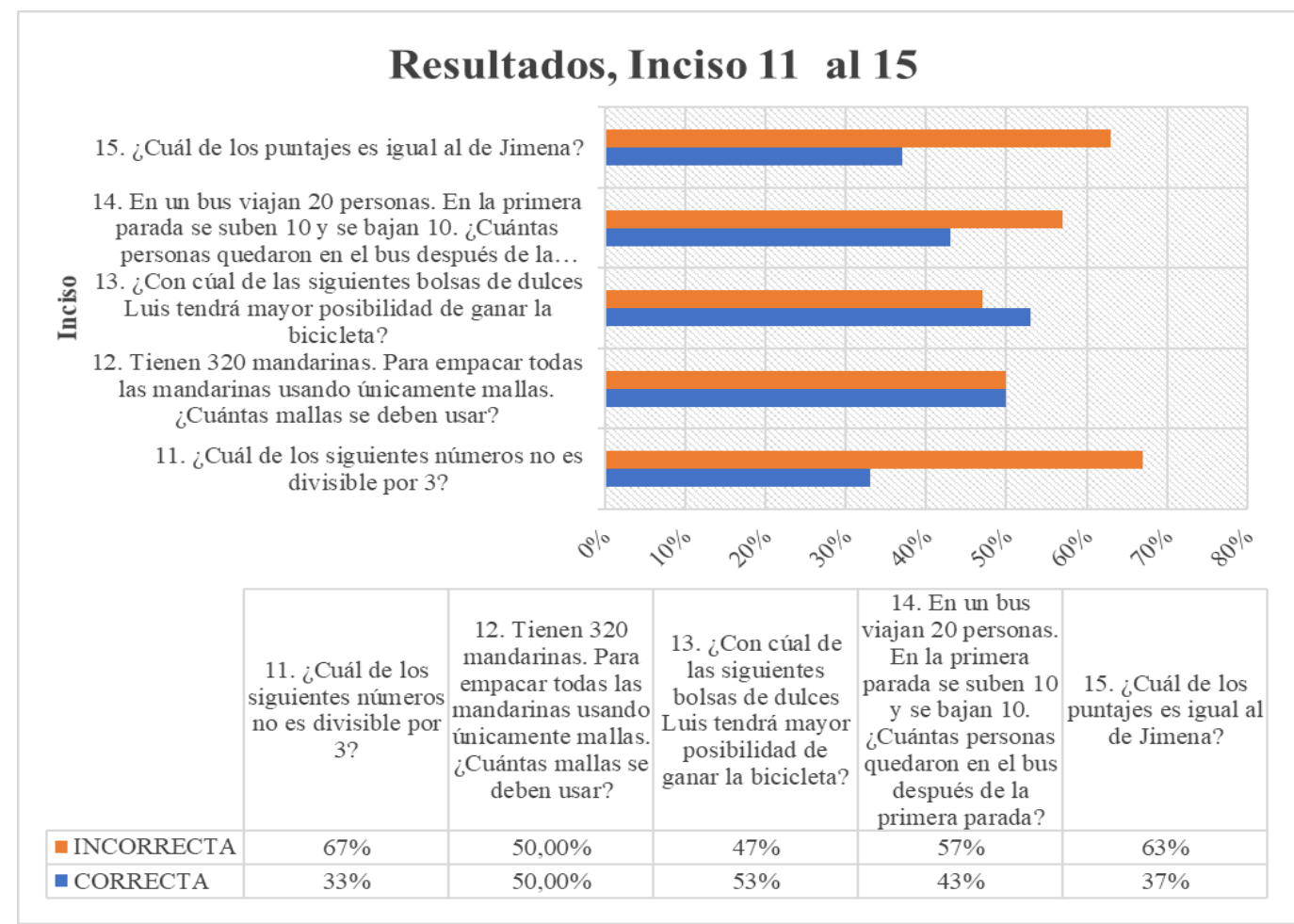

Figura 9. Resultados prueba diagnóstica, incisos del 11 al 15. Fuente: Los autores.

\subsection{Fase final: Prueba diagnóstica luego de haber implementado la estrategia.}

Luego de desarrollada la estrategia pedagógica mediada por las TIC y aplicada durante las clases de matemáticas, se realizó una prueba diagnóstica final para conocer el impacto de dicha herramienta en el aprendizaje de los estudiantes. En las Figuras 10, 11 y $\underline{12}$ se muestran los resultados obtenidos.

A partir de los resultados obtenidos en la prueba diagnóstica, realizada luego de la aplicación de la herramienta didáctica, se pudo evidenciar que más del $40 \%$ de los estudiantes respondió acertadamente todas las preguntas, y que más del $70 \%$ respondió correctamente el $46,7 \%$ de las preguntas, a diferencia de los resultados de la prueba diagnóstica inicial, donde solo una pregunta fue respondida de forma correcta por más del $70 \%$ de los estudiantes; $\mathrm{y}$ el $66,7 \%$ de las preguntas fueron respondidas acertadamente por menos del $50 \%$ de los estudiantes, lo cual permite ratificar que la herramienta didáctica mejoró el indicador de resolución de problemas matemáticos y que la implementación de este tipo de herramientas TIC, hace que haya una correlación positiva con los resultados de los estudiantes.

\subsection{Comparación de resultados}

En el proceso de confrontación de resultados se tomaron los porcentajes de respuestas incorrectas obtenidos en las pruebas diagnósticas inicial y final, los cuales se exponen en la Figura 13.

Luego del respectivo análisis de la Figura 13. Se advierte el avance que han presentado los estudiantes de la institución, evidenciándose una notoria reducción del porcentaje de respuestas incorrectas en la prueba final, lo cual indica efectividad en la aplicación de la herramienta didáctica. Estos resultados son equiparables a los encontrados por Martínez y Gualdrón (2018), donde se evidenció una evolución desde el inicio de la intervención hasta el final, gracias al uso de herramientas didácticas mediadas por las TIC.

Lo anterior, permite afirmar que la herramienta didáctica MATE.TICS generó un impacto positivo en el proceso de enseñanza aprendizaje de las matemáticas en el estudiantado, reflejado en el incremento del porcentaje de respuestas correctas seleccionadas por los estudiantes. 
Martínez Cogollo et al. (2021). Revista Sextante, 24, pp. 2 - 12, 2021.

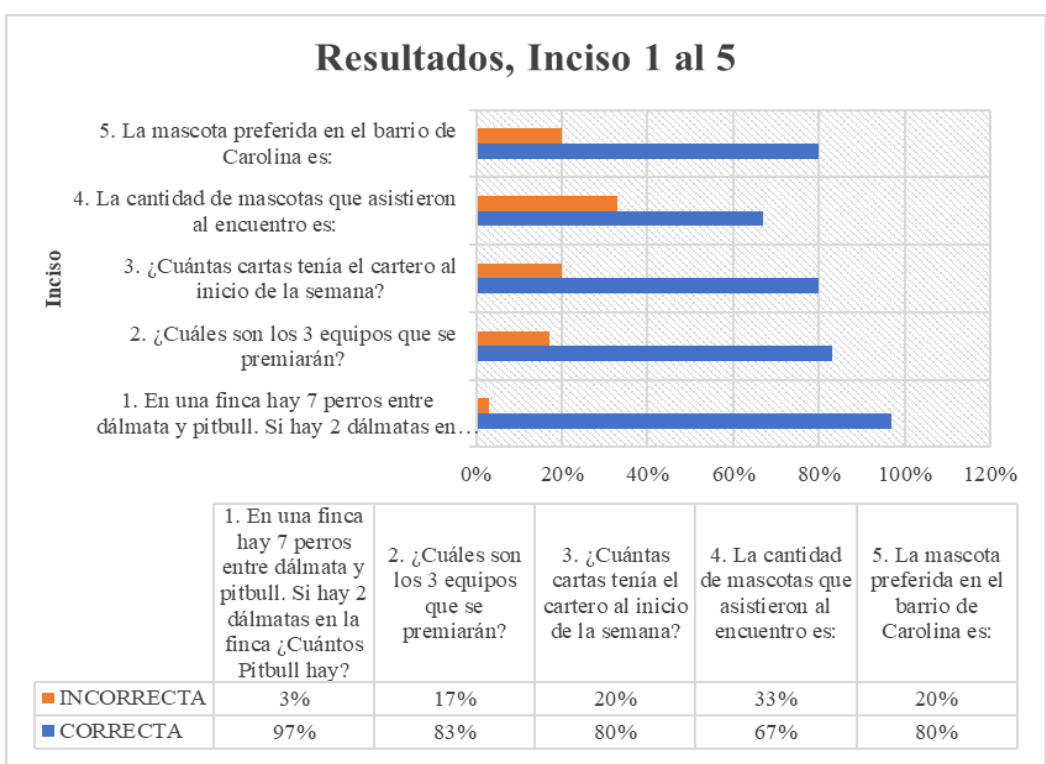

Figura 10. Resultados prueba final, incisos del 1 al 5.

Fuente: Los autores.

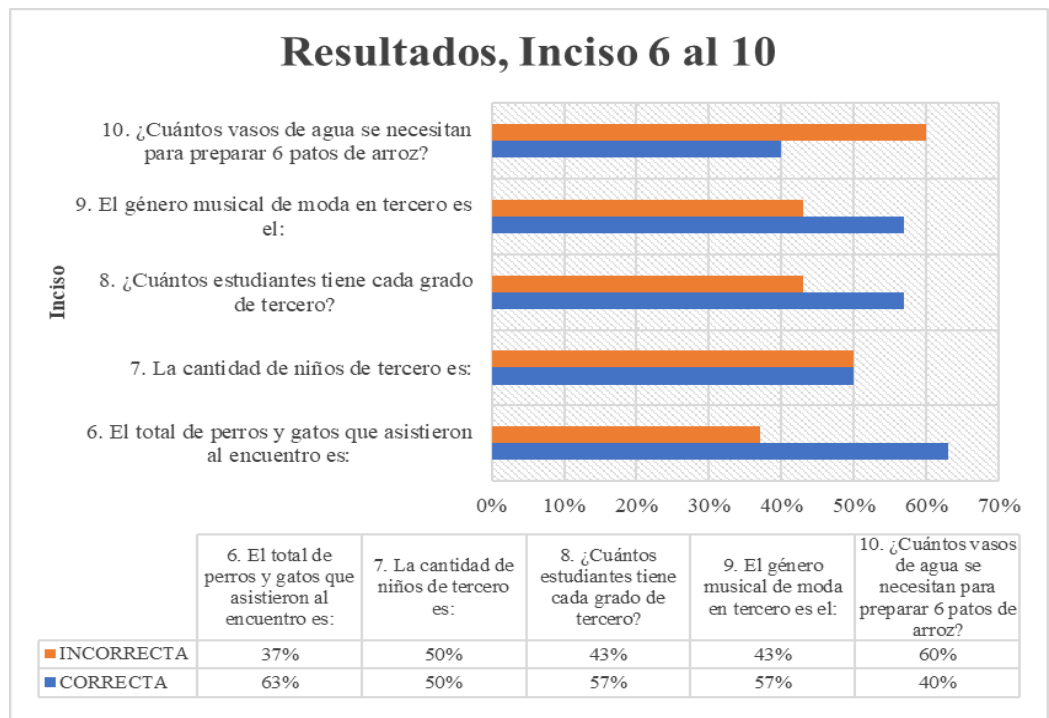

Figura 11. Resultados prueba final, incisos del 6 al 10.

Fuente: Los autores.

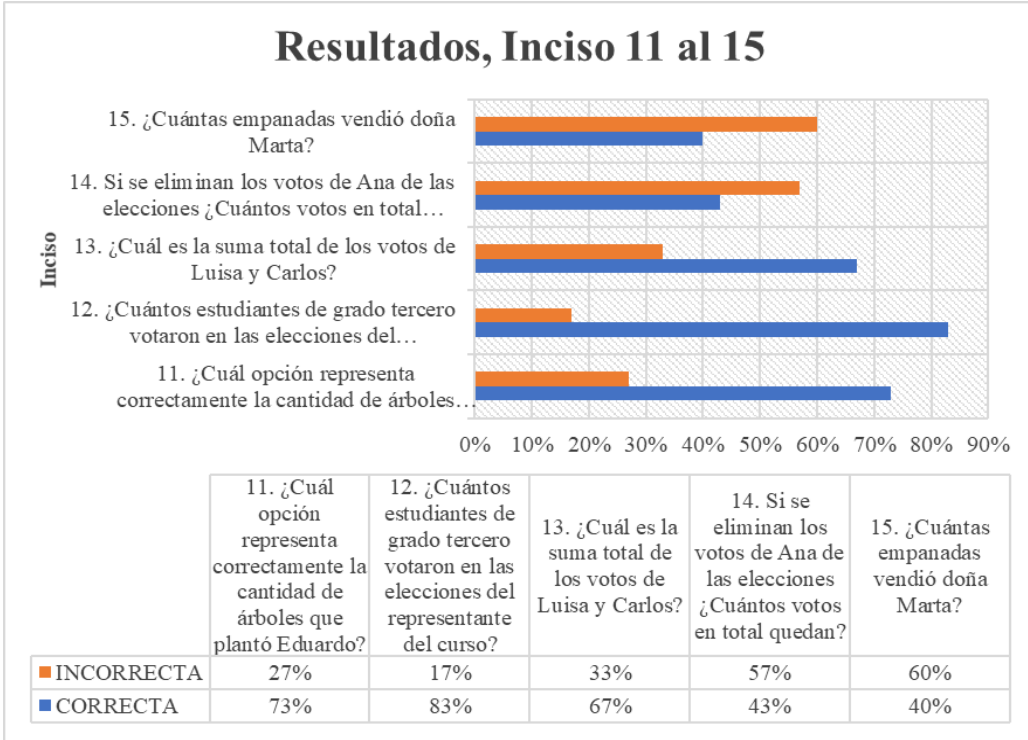

Figura 12. Resultados prueba final, incisos del 11 al 15.

Fuente: Los autores. 


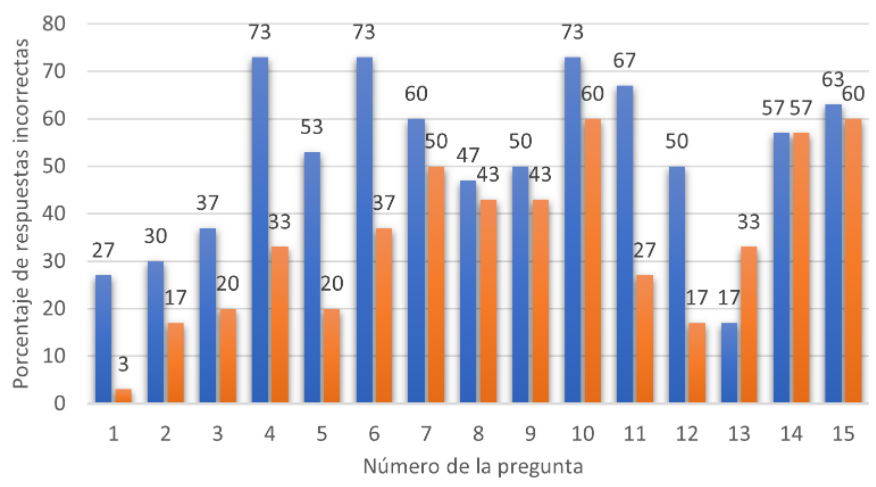

Figura 13. Comparación de resultados de las pruebas inicial y final.

Fuente: Los autores.

Estos resultados son equiparables a los obtenidos por Cuartas, Osorio y Villegas (2015) donde los porcentajes de respuestas correctas pasaron de 30\% y $10 \%$ en la prueba inicial, a $90 \%$ y $100 \%$ en la prueba final, luego de utilizar las herramientas tecnológicas Mazema, Calkulo y Kkuentas, en los ítems correspondientes al pensamiento espacial.

\section{Recomendaciones para trabajos futuros}

Se propone el desarrollo de otras asignaturas con herramientas TIC para aprovechar el interés que despiertan las herramientas tecnológicas en los estudiantes dentro de los diferentes grados y niveles de formación académica.

Por otro lado, cabe recordar que es necesario el uso de las TIC como herramientas pedagógicas, ya que fomentan la capacidad creadora de los estudiantes generando nuevos conocimientos. El uso de estas tecnologías es importante para el desarrollo del proceso enseñanza-aprendizaje, para romper los esquemas de pedagogía tradicional y hacer un buen uso del aula de informática, ampliando su uso fuera de la hora de tecnología e informática, constituyendo una fuente de innumerables trabajos de tipo investigativo.

Finalmente, como parte del compromiso de los docentes y directivos en el proceso de enseñanzaaprendizaje está la apertura y fomento de espacios y escenarios propicios para la promoción del uso de las TIC dentro de los ambientes educativos, en todos los niveles.

\section{Conclusiones}

Se puede concluir que la herramienta didáctica MATE.TICS permite integrar la planeación y el desarrollo de actividades matemáticas a través de la realización de ejercicios que integran juegos interactivos, videos y explicaciones, permitiendo así, un incremento del conocimiento y el interés de los estudiantes de la institución por las matemáticas, motivando a su vez el interés por aprender, y reflejando mejoras en su rendimiento académico.

De igual forma, con la aplicación de la plataforma LSM se evidencia que los estudiantes potenciaron su pensamiento matemático numérico, mejorando su desempeño en esta competencia. Por lo tanto, es posible afirmar que el uso de herramientas didácticas mediadas por las TIC promueve la adquisición del conocimiento de una manera más fácil y rápida, promoviendo así, el aprendizaje significativo.

Finalmente, con esta investigación se logró confirmar que el carácter moderno de las TIC permite que los estudiantes se encuentren familiarizados con esta, para la enseñanzaaprendizaje de las diferentes áreas del conocimiento. Además, deja que se produzca el desarrollo de competencias básicas, fortalecimiento de las capacidades digitales de los estudiantes y su motivación por aprender los temas estudiados.

\section{Referencias}

Cuartas, D., Osorio, C y Villegas. (2015). Uso de las TIC para mejorar el rendimiento en matemática en la escuela nueva. (Tesis de maestría, Universidad Pontificia Bolivariana). https://repository.upb.edu.co/bitstream/handle/2 0.500.11912/2840/T.G-

Dora\%20C.\%20Cuartas\%3B\%20Caludia\%20

M.\%200sorio\%3B\%20Lilian\%20Y\%20Villegas .pdf? sequence $=1$

Hernández, O. (2018). Materiales y recursos tecnológicos para la resolución de problemas de Matemáticas de $3^{\circ}$ y $4^{\circ}$ de Educación Primaria: Geometría y medida. (Tesis de maestría, Universidad de la Laguna). https://riull.ull.es/xmlui/bitstream/handle/915/1 1429/Materiales\%20y\%20recursos\%20tecnolog icos $\% 20$ para $\% 20 l a \% 20$ resolucion $\% 20 \mathrm{de} \% 20 \mathrm{p}$ 
roblemas $\% 20$ de $\% 20$ matematicas $\% 20$ de $\% 203 \%$ C2\%BA\%20y\%204\%C2\%BA\%20de\%20educac ion\%20primaria.\%20Geometria\%20y\%20medi da.pdf? sequence $=1$

Hernández Sampieri, R., Fernández Collado, C., \& Baptista Lucio, M. D. P. (2014). Metodología de la investigación. Mcgraw-hill.

Ibáñez, C. (2007). Un análisis crítico del modelo del triángulo pedagógico. Una propuesta alternativa. Revista mexicana de investigación educativa, 12(32), 435-456.

Martínez-López, L. G., \& Gualdrón-Pinto, E. (2018). Fortalecimiento del pensamiento numérico a través de una intervención mediada con TIC en estudiantes de grado noveno. Revista de Investigación, Desarrollo e Innovación, 9(1), 91-102.

Ministerio de Educación Nacional. (2018). Reporte de Excelencia 2018 (ISCE). https://diae.mineducacion.gov.co/siempre_diael documentos/2018/223686000451.pdf

Ministerio de Tecnologías de la Información y las Comunicaciones. (2009). Ley 1341 de 2009. https://www.mintic.gov.co/portal/inicio/3707:Le y-1341-de-2009

Morales, Y. (2015). Uso de tecnología en la educación: las habilidades básicas del maestro de primaria en la clase de matemática. Revista Tecnología en Marcha, 28(4), 108-121. https://www.scielo.sa.cr/scielo.php? pid=S0379$\underline{39822015000400108 \& \text { script }=\text { sci_arttext }}$

Organización de las Naciones Unidas para la Educación, la Ciencia y la Cultura. (2016). Educación 2030: Declaración de Incheon y Marco de Acción para la realización del Objetivo de Desarrollo Sostenible 4: Garantizar una educación inclusiva y equitativa de calidad $y$ promover oportunidades de aprendizaje permanente para todos. https://unesdoc.unesco.org/ark:/48223/pf00002 45656_spa

Organización para la Cooperación y el Desarrollo Económico- OCDE- (2018). Competencias en Iberoamérica: Análisis de PISA 2015. https://www.educacionyfp.gob.es/inee/dam/jcr:0 al62adc-646c-406a-82754ee09b81e51a/Competencias-en-IberoamericaAnalisis-de-PISA-2015.pdf

Quinde, J y Rodríguez, E. (2018). Aplicación de juegos lógicos para mejorar los logros matemáticos en los estudiantes del segundo grado de primaria de la institución educativa $n^{o}$ 16970 - San José de Lourdes - San Ignacio. (Tesis de maestría, Universidad César Vallejo). http://repositorio.ucv.edu.pe/bitstream/handle/U CV/33347/quinde_pj.pdf? sequence $=1$

Real, M. (2013). Las TIC en el proceso de enseñanza y aprendizaje de las matemáticas. Materiales para el desarrollo curricular de matemáticas de tercero de ESO por competencias.

Siemens, G. (2004). Conectivismo: Una teoría de aprendizaje para la era digital. https://pdfs.semanticscholar.org/05f1/adee 1873 23d66beab226058b23a7416c3517.pdf

Siemens, G. (2006). Knowing knowledge. 